\title{
Microstructure of epitaxial $\mathrm{Mg}_{3} \mathrm{~N}_{2}$ thin films grown by $\mathrm{MBE}$
}

\author{
P. John ${ }^{1}$, P. Vennéguès ${ }^{1}$, H. Rotella ${ }^{1}$, C. Deparis ${ }^{1}$, C. Lichtensteiger ${ }^{2}$ and J. Zúñiga-Pérez ${ }^{1, *}$ \\ ${ }^{1}$ CRHEA, CNRS, UCA, Rue Bernard Gregory, 06560 Valbonne, France \\ ${ }^{2}$ DQMP, University of Geneva, 24 Quai Ernest Ansermet, Geneva 4, 1211, Switzerland \\ * e-mail: jzp@crhea.cnrs.fr
}

\begin{abstract}
The epitaxial growth of $\mathrm{Mg}_{3} \mathrm{~N}_{2}$ thin films by molecular beam epitaxy has been recently achieved. This work presents the structural properties of the films, including grains sizes and lattice rotations, as assessed by $x$-ray diffraction and transmission electron microscopy. The films' microstructure consists of well-aligned columnar grains $10 \mathrm{~nm}$ in diameter that nucleate at the film/substrate interface and that display a significant column twist, in the order of $2.5^{\circ}$. As growth proceeds, tilted and twisted mosaic blocs overgrow these columns, as observed in to many other epitaxial semiconductors. Yet, the rocking curves on symmetric reflections display extremely narrow peaks ( 50 arcseconds), revealing a long-range spatial correlation between structural defects that should not be mistakenly considered as a proof of high crystalline quality.
\end{abstract}




\section{INTRODUCTION}

Group III-nitride semiconductors are widely applied in products of daily life, such as LEDs ${ }^{1}$, lasers ${ }^{2}$ and transistors ${ }^{3}$. As a consequence, there has been a long and strong interest in establishing their fundamental physical properties, in controlling their crystal growth and in developing new device structures ${ }^{4}$. On the contrary, there are no commercial devices based on group II-nitrides, their growth is not well controlled and some of their basic properties are not yet well determined. Among this family of materials, $\mathrm{Mg}_{3} \mathrm{~N}_{2}$ seems an interesting candidate for device applications due to its relatively large and direct bandgap of 2.8 to $2.9 \mathrm{eV}$ at room temperature ${ }^{5-7}$. Additionally, alloying $\mathrm{Mg}_{3} \mathrm{~N}_{2}$ with $\mathrm{Zn}_{3} \mathrm{~N}_{2}$ seems a promising way to address solar energy applications, given the possibility to tune the optical bandgap down to about $1.4 \mathrm{eV}$ or lower ${ }^{8}$. Nevertheless, the epitaxial growth of $\mathrm{Mg}_{3} \mathrm{~N}_{2}$ crystals has been established only recently ${ }^{7}$, previous reports dealing mainly with $\mathrm{Mg}_{3} \mathrm{~N}_{2}$ micro-powders, which prevented the development of $\mathrm{Mg}_{3} \mathrm{~N}_{2}$-based devices.

Even though a first attempt of growing epitaxial $\mathrm{Mg}_{3} \mathrm{~N}_{2}(100)$ on $\mathrm{MgO}(100)$ by molecular beam epitaxy (MBE) was reported in $2018^{9}$, detailed structural analysis of these layers was not possible due to low crystallinity. More recently, we demonstrated the possibility of achieving epitaxial $\mathrm{Mg}_{3} \mathrm{~N}_{2}$ by plasmaassisted MBE and the tuning of the crystallographic orientation depending on the $\mathrm{Mg}_{3} \mathrm{~N}_{2}$ growth conditions ${ }^{7}$. Indeed, by changing the $\mathrm{Mg} / \mathrm{N}$ ratio it is possible to switch the film orientation from (100) to (111). Further, thanks to a capping layer of $\mathrm{MgO}$ grown in the $\mathrm{MBE}$ chamber right after the $\mathrm{Mg}_{3} \mathrm{~N}_{2}$ growth, we prevent the otherwise irreversible decomposition of the $\mathrm{Mg}_{3} \mathrm{~N}_{2}$ films in $\mathrm{air}^{7,9}$. This ingredient is essential to perform deep structural $x$-ray diffraction investigations, which take much longer than the $\mathrm{Mg}_{3} \mathrm{~N}_{2}$ lifetime in $\mathrm{air}^{7}$.

X-ray diffraction (XRD) enables detailed structural analysis of crystalline thin films for a large range of materials in a non-destructive manner. Lattice parameters as well as crystalline quality can be accurately measured using this technique. The latter one is especially important when dealing with heteroepitaxial growth, where the density of introduced defects can be particularly large due to 
differences in crystal structure, orientation, lattice parameters and/or chemical composition of substrate and film. These mismatches lead to strain in the growing films, which is released by the formation of defects such as dislocations or low-angle grain boundaries. The presence of defects gives rise to local rotations and distortions of the crystal planes close to them, leading to a partial or complete loss of $\mathrm{x}$-ray scattering coherence. Lattice rotations around an in-plane axis are commonly referred to as tilt, whereas lattice rotations around an out-of-plane axis are referred to as twist, being two of the three ingredients usually employed to describe epitaxial films as mosaic crystals (together with the size of the mosaic blocks) ${ }^{10}$. Consequently, line widths of diffraction rocking curves increase as a function of the defect density. However, if the position and, eventually, the orientation of the defects are correlated, coherent scattering can be preserved even in the presence of large defect densities, leading to extremely narrow line widths in transverse $\omega$ scans $^{11-25}$. While XRD gives a global view of the crystal's microstructure and lattice parameters, transmission electron microscopy (TEM) enables a local insight into the film structure that allows the identification of lattice defects and the determination of their spatial arrangement. Unfortunately, TEM is a destructive technique that requires time-consuming sample preparation. This becomes a strong handicap when dealing with airsensitive samples like $\mathrm{Mg}_{3} \mathrm{~N}_{2}$, given that their preparation and latter transfer to the electron microscope have to be done under vacuum to prevent sample degradation.

In this contribution, the line shapes of XRD peaks of $\mathrm{Mg}_{3} \mathrm{~N}_{2}$ thin films with different thicknesses have been thoroughly investigated in the context of lattice distortions. XRD rocking curves of symmetric reflections, exhibiting concomitantly a narrow (coherent) scattering peak as well as broad (diffuse) scattering one, were used to extract the thickness range in which either finite-size effects (with a lateral grain size of around $10 \mathrm{~nm}$ ) or lattice rotations (tilt) dominate. Analysis of asymmetric reflections allowed the evaluation of the twist. Despite the high sensitivity of $\mathrm{Mg}_{3} \mathrm{~N}_{2}$ to ambient conditions (humidity), we were able to investigate the films by TEM for the first time, completing the results obtained from XRD and illustrating the nano/microstructure of the $\mathrm{Mg}_{3} \mathrm{~N}_{2}$. 


\section{EXPERIMENTAL}

A series of epitaxial $\mathrm{Mg}_{3} \mathrm{~N}_{2}$ (111) films with different thicknesses was grown on $\mathrm{MgO}(100)$ substrates by plasma-assisted MBE using a Riber EPINEAT system. The growth chamber is equipped with an effusion cell for elemental $\mathrm{Mg}(99.9999 \%)$ and a radio-frequency $(13.56 \mathrm{MHz})$ plasma cell for atomic nitrogen which was obtained by energizing nitrogen gas $(99.9999 \%)$ with a plasma power of $360 \mathrm{~W}$. The growth temperature for the thickness series was $400{ }^{\circ} \mathrm{C}$ (see Sample E in reference [7] for more details). The $10 \times 20 \mathrm{~mm}^{2} \mathrm{MgO}$ substrates were prepared prior to growth by rapid thermal annealing for $2 \mathrm{~min}$ at $1150{ }^{\circ} \mathrm{C}$ under oxygen to obtain atomically flat surfaces. As-grown $\mathrm{Mg}_{3} \mathrm{~N}_{2}$ films were capped with ca. $50 \mathrm{~nm} \mathrm{MgO}$ in the MBE chamber in order to prevent the fast decomposition of $\mathrm{Mg}_{3} \mathrm{~N}_{2}$ to $\mathrm{Mg}(\mathrm{OH})_{2}$ and $\mathrm{NH}_{3}$, which is observed in ambient air ${ }^{7,26-28}$.

XRD experiments were performed using a Panalytical X'Pert PRO MRD diffractometer equipped with a $\mathrm{Cu}$ anode and a Ge (220) asymmetrical four-crystal monochromator providing $\mathrm{Cu}_{\alpha 1}$ radiation (1.54056 ̊̊). An x-ray mirror leads to a parallel beam with a divergence of less than $0.05^{\circ}$. Highresolution rocking curves (so-called transverse or $\omega$ scans) and longitudinal $2 \theta / \omega$ scans were measured on symmetric $\mathrm{Mg}_{3} \mathrm{~N}_{2}$ (222) reflections using the triple axis configuration with a Ge analyzer crystal in which the diffracted beam undergoes three (220) reflections before entering the detector. Rocking curves of asymmetric as well as $\mathrm{Mg}_{3} \mathrm{~N}_{2}$ (444) reflections were measured using a fully open detector or with a $1 / 4^{\circ}$ slit in the diffraction path.

The nanostructure of selected samples was further investigated using TEM. Sample preparation by focus ion beam sectioning using a FEI Helios 600 NanoLab dual beam instrument (CP2M, Marseille, France) was performed for observations along the $\mathrm{MgO}$ [011] substrate direction. One sample of the thickness series ( $302 \mathrm{~nm} \mathrm{Mg} 3 \mathrm{~N}_{2}$, grown at $400{ }^{\circ} \mathrm{C}$ ) is compared to a $97 \mathrm{~nm}$ thick sample grown at higher substrate temperature $\left(460{ }^{\circ} \mathrm{C}\right.$, see sample $\mathrm{F}$ in reference [7] for more details). Further, one plan view specimen of a $90 \mathrm{~nm}$ thick sample of the thickness series was prepared. Ga ions from a liquid metal source were accelerated with $30 \mathrm{kV}$ onto the samples in order to prepare the thin specimens by ion 
beam milling. Final thinning was performed at $1 \mathrm{kV}$ to minimize sample damage caused by the ion beam. After preparation, the samples were transported in a vacuum container to the TEM and then investigated using a FEI Titan 80-300 objective-corrected microscope, operated at $200 \mathrm{kV}$ (CP2M, Marseille, France).

\section{RESULTS}

\section{A. XRD of symmetric reflections}

A series of six $\mathrm{Mg}_{3} \mathrm{~N}_{2}$ (111) samples having different film thicknesses was investigated by XRD. Figure 1 (a) shows the high-resolution $2 \theta / \omega$ scans across the $\mathrm{Mg}_{3} \mathrm{~N}_{2}(222)$ reflection for all samples. The curves were normalized and then vertically shifted for clarity. Finite thickness interference fringes are clearly visible for the films up to $131 \mathrm{~nm}$, demonstrating vertical $\mathrm{x}$-ray coherence. The fringes vanish for thicker samples, indicating an increased surface roughness and/or decrease of $\mathrm{x}$-ray coherence due to a degradation of crystalline quality. Assuming a perfect crystalline coherence without any roughness, the theoretical $2 \theta / \omega$ diffraction pattern is given by ${ }^{29}$

$$
I(\theta) \propto \frac{\sin ^{2}\left(2 \pi n d_{222} \sin \theta / \lambda\right)}{\sin ^{2}\left(2 \pi d_{222} \sin \theta / \lambda\right)}
$$

where $d_{222}$ is the interplanar distance of $\mathrm{Mg}_{3} \mathrm{~N}_{2}$ (222) planes, $n$ is the total number of unit cells in the growth direction, $\theta$ is the diffraction angle and $\lambda$ is the $x$-ray wavelength. The resulting thicknesses obtained by simulating the XRD patterns with Equation (1) are indicated in Figure 1 and are consistent with $\mathrm{x}$-ray reflectivity (XRR) measurements (see Figure S1 of the supplemental material). Note that the thicknesses of the thickest layers were obtained by extrapolating the growth rate of the thinner ones.

The slight shift to higher $2 \theta$ angles with increasing sample thickness indicates a decrease in $d_{222}$, which can be generally interpreted as a change in strain and/or chemical composition of the films. The lattice mismatch between layer and substrate necessarily leads to strain in the $\mathrm{Mg}_{3} \mathrm{~N}_{2}$ films, especially at the beginning of the growth, as commonly observed in heteroepitaxy. Previous reflection high energy 
electron diffraction (RHEED) investigations on epitaxial $\mathrm{Mg}_{3} \mathrm{~N}_{2}$ thin films revealed a compressive inplane strain at early growth stages ${ }^{7}$, as expected from the epitaxial relationship between film and substrate (lattice mismatch $+2.3 \%$ along $\mathrm{Mg}_{3} \mathrm{~N}_{2}[11 \overline{2}]$ and $+18.2 \%$ along $\mathrm{Mg}_{3} \mathrm{~N}_{2}$ [11̄0], calculated with a domain phase matching condition of two $\mathrm{MgO}$ unit cells for one $\mathrm{Mg}_{3} \mathrm{~N}_{2}$ cell). This is in agreement with the observation of asymmetric line shapes of the diffraction peaks, which indicate an inhomogeneous strain across the sample thickness. By introducing a $d_{222}$ in Equation (1) that varies with thickness, it is possible to reproduce this asymmetric line shape and quantify the change in lattice spacing with thickness. Lichtensteiger ${ }^{30}$ developed a software for fitting $2 \theta / \omega$ line profiles in XRD in which the lattice spacing can change gradually as a function of film thickness with the following functional dependence

$$
d_{222}=\mathrm{A} e^{-N / B}+C
$$

where $N$ is the running unit cell number and $A, B$ and $C$ are fitting parameters. Using Equation (2) allowed us to reproduce the asymmetry observed in the experimental diffraction curves, as shown by the dotted lines in Figure 1(a), where the inset shows the resulting (222) lattice spacings as a function of film thickness obtained from the simulations. The variation of the interplanar lattice distances as a function of thickness indicates an in-plane compressive strain close to the film-substrate interface, which evolves into a tensile strain for thicknesses larger than 70 unit cells (ca. $20 \mathrm{~nm}$ ). This residual tensile strain might be introduced by grain coalescence, as observed in many other epitaxial systems ${ }^{31-}$ ${ }^{33}$. Note that the additional interference fringes in the simulation of the thinnest ( $\left.15 \mathrm{~nm}\right) \mathrm{Mg}_{3} \mathrm{~N}_{2}$ film are due to the $\sim 50 \mathrm{~nm} \mathrm{MgO}$ capping layer, which is included in the model.

A pure variation of the lattice parameter is, however, not sufficient to obtain a perfect agreement between the simulated and the experimental curves for the thicker films. The reason for this lies most probably in increased roughness and/or decreased crystallinity, as will be discussed in the following.

The high-resolution transverse $\omega$ scans of the same sample series around the symmetric $\mathrm{Mg}_{3} \mathrm{~N}_{2}(222)$ reflections are shown in Figure 1 (b). All curves exhibit two contributions, a narrow (coherent) component and a broad (diffuse) one. As shown in the inset of Figure 1(b), the transverse $\omega$ scan of 
the thinnest $(15 \mathrm{~nm}) \mathrm{Mg}_{3} \mathrm{~N}_{2}$ film is dominated by an extremely narrow contribution that has approximately the same linewidth as the symmetric (200) reflection of the $\mathrm{MgO}$ substrate (measured at a different $2 \theta$ angle). At this stage, we assume that the coherent $x$-ray scattering phenomenon leading to this peak is associated with a regular distribution of some crystalline defect displaying very long correlation distances, as observed previously in other material systems ${ }^{11-25}$.

Interestingly, while an increase of the overall diffuse scattering intensity with respect to the coherent one is observed as a function of sample thickness, the integrated intensity of the coherent peak itself remains roughly constant. This observation points towards its origin being located close to the filmsubstrate interface, with the initially correlated defects being overgrown by a disordered $\mathrm{Mg}_{3} \mathrm{~N}_{2}$ displaying correlations at much smaller spatial scales. Such line shapes have been reported previously in various heteroepitaxial systems and are usually interpreted as originating from different types of correlated defects, including threading dislocations ${ }^{11-16}$, stacking faults ${ }^{17}$, misfit dislocations located at the film-substrate interface ${ }^{16,18-24}$, misorientations ${ }^{12,22,23}$ or grain boundaries ${ }^{17,25}$. Consistent with these previous reports, the intensity of the coherent peak decreases significantly when going from the (222) to the (444) reflection, as observed in Figure 2.

If we now focus our attention on the broad, diffuse component, we should first recall that as early as 1953 Williamson and Hall ${ }^{34}$ analyzed the line widths of consecutive diffractions orders (so-called Williamson-Hall plot) as a means to separate vertical finite-size and strain contributions to the broadening in $2 \theta / \omega$ scans. A similar procedure can be introduced to analyze transverse $\omega$ scans of symmetric reflections, enabling this time to separate in-plane finite-size effects from lattice rotations (around an axis contained parallel to the sample surface, i.e. tilt broadening). Miceli et al. ${ }^{22,23}$ observed that for finite-size broadening, caused by e.g. small crystallites, the line width (in units of $Q_{x}$ ) appears constant as a function of the length of the scattering vector $\boldsymbol{Q}$ (i.e. reflection order). On the other hand, for broadening due to lattice rotations (tilt), the line width is proportional to the length of $\boldsymbol{Q}$. Such an analysis is presented for our films in Figure 2 in logarithmic scale, where the rocking curves of the (222) 
and (444) reflections of the $69 \mathrm{~nm}$ and $302 \mathrm{~nm} \mathrm{Mg} \mathrm{N}_{2}$ films are displayed. Figure 2(a) shows the reflections for the $69 \mathrm{~nm}$ layer in units of $Q_{x}$. The two-component line shape is clearly visible around both reflections and, as already stated, the intensity of the narrow contribution decreases significantly when going from the (222) to the (444) reflection. Most important, rescaling the diffuse scattering of the (444) reflection using a multiplicative factor reveals that the line width of the diffuse scattering is constant as a function of transverse scattering vector $Q_{x}$, indicating that finite-size effects (e.g. limited grain sizes) are the main origin of line broadening. In this case, the full width at half maximum (FWHM) of the diffuse scattering, $\Delta Q_{x}$, is inversely proportional to the length $l$ causing the peak broadening, which can be interpreted as the lateral grain size $\left(l=2 \pi / \Delta Q_{x}\right)$ and is $l \approx 13 \mathrm{~nm}$ in Figure 2(a).

Figure 2(b) shows the rocking curves of the (222) and (444) reflections of the $302 \mathrm{~nm}$-thick $\mathrm{Mg}_{3} \mathrm{~N}_{2}$ film, this time as a function of angular units $\omega$. The line shape of the curves is similar to the ones shown in Figure 2(a). However, rescaling by a multiplicative factor $k$ reveals that the FWHM of the diffuse scattering is in this case constant in angular units and, hence, increases as a function of scattering vector as seen in the inset of Figure 2(b), where the same curves are plotted in units of $Q_{x}$. This demonstrates clearly that lattice rotations are the main origin of the line broadening for the thicker samples, giving rise to a two-layer film structure. Note that these peak analyses were done with an open detector equipped with a $1 / 4^{\circ}$ slit in the diffraction path in order to have sufficient intensity to measure the (444) reflection, while still being able to clearly separate the coherent peak from the diffuse one, which is necessary to deconvolute both contributions. Indeed, it should be mentioned that the FWHM of the narrow, coherent peak in Figure 2(b) increases from 100 arcsec, via 196 arcsec, to 238 arcsec when changing the detector from a crystal analyzer, via $1 / 4^{\circ}$ slit, to a completely open configuration. On the other hand, the FWHM of the diffuse contribution stays almost constant $\left(1.3 \pm 0.1^{\circ}\right)$ as a function of the detector configuration (see Figure S2 of the supplemental material). This justifies why we can analyze the diffuse scattering while using a $1 / 4^{\circ}$ slit configuration. 
In order to better identify the critical thickness at which the transition from a finite-size to a rotationlimited broadening occurs, we calculated the difference of the diffuse scattering curves between the (222) and (444) reflections as a function of $\omega$ as well as $Q_{x}$

$$
\Delta=\frac{1}{N} \frac{\sum_{i=1}^{N}\left(I_{222}^{\text {diff }}-k I_{444}^{\text {diff }}\right)^{2}}{\left(I_{\max }^{\text {diff }}\right)^{2}}
$$

where $I_{222}^{\text {diff }}$ and $I_{444}^{\text {diff }}$ are the diffuse scattering intensities of the (222) and (444) reflection, respectively, and $N$ is the number of measurement points. By minimizing Equation (3), the differences of the (222) and (444) curves as a function of $\omega$ or of $Q_{x}$ can be extracted, as well as the multiplicative factor $k$ necessary to rescale the diffuse scattering of the (444) reflection (this factor is used to plot the curves in Figure 2). The result is normalized by the maximum of the diffuse scattering intensity $I_{\max }^{\text {diff }}$, which allows to quantitatively compare this goodness parameter $\Delta$ among different samples. If $\Delta$ is smaller when calculated in units of $Q_{x}$ (i.e. the agreement between the curves is better when plotted as a function of $Q_{x}$ ), the peak broadening is mainly caused by finite-size effects, i.e. limited grain sizes. In the opposite case, if $\Delta$ is smaller when calculated in units of $\omega$, lattice rotations are the main origin of the peak broadening. In Figure 3(a), the goodness parameter is plotted as a function of $\mathrm{Mg}_{3} \mathrm{~N}_{2}$ film thickness. For the two thinnest $\mathrm{Mg}_{3} \mathrm{~N}_{2}$ films, $15 \mathrm{~nm}$ and $69 \mathrm{~nm}, \Delta$ is smaller when calculated in units of $Q_{x}$, confirming again that the diffuse x-ray scattering is dominated by finite-size effects, with a lateral grain size of 9 to $13 \mathrm{~nm}$ (depending on the sample). As the thickness of the films increases, $\Delta$ becomes large when calculated in units of $Q_{x}$, showing that the line width is not anymore constant as a function of scattering order. Indeed, all samples that are thicker than $69 \mathrm{~nm}$ show a smaller $\Delta$ when calculated in angular units $\omega$, confirming that lattice rotations are the principal source of peak broadening for these samples. The crossing of the two behaviors lies between 70 to $90 \mathrm{~nm}$, which is the critical thickness where the transition takes place.

The origin of line broadening can be extracted not only by comparing line shapes of consecutive diffraction orders, but also by analyzing the tails of the diffraction curves for sufficiently large $Q_{x}$. Kaganer et $a l .{ }^{15}$ developed a model to fit tails of $\mathrm{x}$-ray diffraction rocking curve profiles. More 
specifically, when measured with a fully open detector, they attributed a $Q_{x}^{-2}$ decay to finite sizes as the origin of peak broadening, while a $Q_{x}^{-3}$ dependence was shown to originate from lattice defects, in their case randomly distributed threading dislocations. Figure 3(b) shows the (444) diffraction rocking curves of our sample series measured with an open detector in a log-log scale. This representation has the advantage that curves following a power law become straight lines, where slopes of -2 and -3 indicate $Q_{x}^{-2}$ and $Q_{x}^{-3}$ dependencies, respectively. For the thinnest $\mathrm{Mg}_{3} \mathrm{~N}_{2}$ layers, the tails of the rocking curve (at around $Q_{x}=1 \mathrm{~nm}^{-1}$ ) exhibit a slope of -2 and, hence, $Q_{x}^{-2}$ dependence, pointing towards finite-size effects as the main origin of peak broadening. For thicker samples, a continuous increase of the tail slope is observed until a value of -3 is reached at a thickness of $131 \mathrm{~nm}$. Interestingly, the slope of our curves increases even further as the film thickness increases, as seen for the thickest sample (547 $\mathrm{nm}$ ), showing a $Q_{x}^{-4}$ dependence. This slope, not explained in the model of Kaganer et al., may indicate other mechanisms operating for thicker samples. To unravel them and draw a complete picture of the $\mathrm{Mg}_{3} \mathrm{~N}_{2}$ films microstructure we have performed TEM measurements both on cross section and on plan view specimens.

\section{B. TEM analysis}

The method of choice to examine the nature and spatial distribution of lattice defects in crystals is TEM, as it gives a direct local insight into the film structure. In this sense it is thus complementary to XRD. Cross section TEM was performed on the $302 \mathrm{~nm}$-thick $\mathrm{Mg}_{3} \mathrm{~N}_{2}$ sample of the thickness series as well as on a thinner, $97 \mathrm{~nm}$-thick sample investigated previously by $\mathrm{XRD}^{7}$.

Figure 4(a) shows a TEM multi-beam micrograph along an $\mathrm{MgO}\langle 011\rangle$ zone axis of the entire film structure for the $302 \mathrm{~nm}$-thick sample. The difference in contrast between the MgO substrate and the MgO capping layer is due to a difference in crystallinity. The MgO capping appears to be polycrystalline in contrast to the MgO substrate, being in agreement with RHEED observations (see Figure S3(a) of the supplemental material). Still, the capping layer is dense and there is no interdiffusion visible with the underlying $\mathrm{Mg}_{3} \mathrm{~N}_{2}$ (HRTEM micrograph shown in Figure $\mathrm{S} 3(\mathrm{~b})$ of the supplemental material). This 
confirms the in-situ $\mathrm{MgO}$ deposition as an adequate means to protect the underlying $\mathrm{Mg}_{3} \mathrm{~N}_{2}$ film. In the $\mathrm{Mg}_{3} \mathrm{~N}_{2}$ film itself, a columnar structure is clearly observed at initial growth stages, i.e. close to the film-substrate interface, with a lateral grain size in the order of $10 \mathrm{~nm}$, being in perfect agreement with the results obtained by XRD. The film-substrate interface appears to be abrupt and the film is clearly crystalline, as confirmed by the high-resolution TEM micrograph shown in Figure 4(b). Note that Moiré patterns are present in the high-resolution image of the columnar $\mathrm{Mg}_{3} \mathrm{~N}_{2}$ film due to the relatively high specimen thickness (several tens of nanometers) with respect to the column diameter, leading to a superposition of several $\mathrm{Mg}_{3} \mathrm{~N}_{2}$ grains within the thickness of the TEM lamella. As the thickness increases, the columnar structure becomes less clear, which is accompanied by a degradation of the film crystallinity, as signalized by the increased brightness in Figure 4(a). Fourier transforms of highresolution images (the high-resolution images are not displayed) taken at the positions indicated by black squares in Figure 4(a) confirm the decrease in crystallinity: Inset A, which shows the Fourier transform close to the film-substrate interface, is in agreement with the expected diffraction pattern (simulation shown in Figure 5(a)) and clearly results from a crystalline film. As the thickness increases, circular arcs appear in the Fourier transform indicating an increasing crystalline disorder, as shown in Inset B. Finally, these arcs dominate the Fourier transform when approaching the interface between the $\mathrm{Mg}_{3} \mathrm{~N}_{2}$ and the $\mathrm{MgO}$ capping layer (Insets $\mathrm{C}$ and $\mathrm{D}$ ), demonstrating strong tilt in the $\mathrm{Mg}_{3} \mathrm{~N}_{2}$ film and being again consistent with RHEED observations (as shown in Figure $\$ 4$ of the supplemental material).

The Fourier transform of Inset A as well as selected area electron diffraction (SAED) experiments of the cross section specimen shown in Figure 5(a) prove the presence of four epitaxial twin domains. This multiplicity of domains is expected in this epitaxial system, due to the unequal rotational symmetry of the $\mathrm{Mg}_{3} \mathrm{~N}_{2}$ (111) films and the $\mathrm{MgO}(100)$ substrates $^{35}$. The epitaxial relationships identified previously by XRD and RHEED ${ }^{7}$ are now confirmed by TEM:

[11̄0] $\mathrm{Mg}_{3} \mathrm{~N}_{2} \| \mid[01 \overline{1}] \mathrm{MgO}$

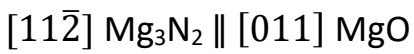


plus the three twins obtained by rotating the $\mathrm{Mg}_{3} \mathrm{~N}_{2}$ structure by $90^{\circ}, 180^{\circ}$ and $270^{\circ}$ around the $\mathrm{Mg}_{3} \mathrm{~N}_{2}$ [111] direction normal to the surface. A schematic representation of the arrangement of the four twin domains on the $\mathrm{MgO}$ substrate is presented in Figure 5(b). The simulated diffraction pattern in Figure 5(a) was obtained by the superposition of the diffraction patterns of the four individual twin domains, where the color of the diffraction pattern indicates diffraction of the respective twin in Figure 5(b) and grey dots indicate diffraction from the $\mathrm{MgO}$ substrate (see supplemental material in reference [7]).

To identify the nature of the columnar grains present in the initial growth stages, we performed two beam observations using $g=(044)$. As shown in the dark field image in Figure $5(c)$, only some of the columns diffract and appear bright when selecting the $g=(044)$ for one specific twin domain family (e.g. Twin I in Figure 5(a) and (b)). This result shows clearly that the columns correspond to the different twin domains present in the film. Furthermore, an average column height of about $70 \mathrm{~nm}$ is observed, consistent with the XRD observations discussed above that pointed towards a change in structural properties around $70-90 \mathrm{~nm}$ (from finite-size limited to tilt limited structural quality). Beyond this thickness, there is less bright contrast, indicating a loss of diffraction conditions due to increased film twist. Interestingly, we are able to control the column height by changing the growth parameters: Figure 5(d) shows the same analysis on a sample grown at higher substrate temperature. The columns show the same width of about $10 \mathrm{~nm}$, but they stay intact and diffract over the entire film thickness of $97 \mathrm{~nm}$. Further, by selecting the $\mathrm{g}=(044)$ of Twin II (see Figure 5(a) and (b)), we obtain bright contrast from complementary columns, as presented in Figure 5(e), although it was not possible to obtain perfect complementary images due to the high specimen thickness with respect to the column diameter, as discussed earlier.

The microstructure of our epitaxial $\mathrm{Mg}_{3} \mathrm{~N}_{2}$ films, with two-component XRD rocking curves and a columnar structure, resembles that observed by Heinke et al. ${ }^{25}$ on c-plane GaN grown on c-plane sapphire. Their TEM investigation revealed a columnar structure as well, with an average column size 
of $24 \mathrm{~nm}$, where the columns were identified as GaN inversion domains with reversed polarity. In this case, the narrow coherent peak was associated to a regular distribution of inversion domain boundaries. We can therefore assume that the regular distribution of twin boundaries, present between columns of equal lateral size in our samples, is the origin of the narrow peaks observed in the XRD rocking curves.

\section{XRD of asymmetric reflections}

As discussed in Section III A and III B, the x-ray scattering characteristics of the thin films of our series are resulting from $\mathrm{Mg}_{3} \mathrm{~N}_{2}$ columnar grains with a width of around $10 \mathrm{~nm}$ and an average height of $70 \mathrm{~nm}$, overgrown by $\mathrm{Mg}_{3} \mathrm{~N}_{2}$ whose misorientation (tilt) increases dramatically with thickness. The quantitative information on the out-of pane disorder (tilt) has been obtained thanks to the measurement of symmetric reflections.

Measuring asymmetric reflections (skew geometry) coming from planes with inclination angles between $0^{\circ}$ and $90^{\circ}$ to the surface has the advantage that peak broadening contains information of both out-of-plane and in-plane disorder (tilt and twist, respectively). However, extracting this information from asymmetric reflections is only possible by measuring several of them in combination with subsequent modelling. Lee et al. ${ }^{36}$ developed a model that allowed them to evaluate tilt/twist generated by threading dislocations in GaN, AIN and AIGaN thin films grown heteroepitaxially on the basal planes of sapphire or SiC. Within this model, the FWHMs of diffraction planes are given by

$$
\Gamma_{h k l}=\sqrt{\left(\Gamma_{y} \cos \chi\right)^{2}+\left(\Gamma_{z} \sin \chi\right)^{2}+(2 \pi / L)^{2} / Q_{h k l}^{2}}
$$

where $\Gamma_{y}$ and $\Gamma_{z}$ are the lattice rotations around an in-plane (i.e. tilt) and the out-of-plane (twist) axis,

respectively, $Q_{h k l}=\frac{4 \pi}{\lambda} \sin \theta$ is the magnitude of the scattering vector $\boldsymbol{Q}$ of the $(h k l)$ reflection and $\chi$ is the angle between the $(h k l)$ plane and the film surface. The effective coherence length $L$ is composed of the lateral grain size $l$ and the vertical grain size $d$, correcting the broadening coming from finite grain sizes. 
The measured FWHMs (in angular units) of rocking curves of various asymmetric reflections, as a function of the inclination angle $\chi$ with respect to the $\mathrm{Mg}_{3} \mathrm{~N}_{2}$ (111) planes, are presented in Figure 6(a) for a sample with rocking curves on symmetric reflections already dominated by tilt. A clear increase of FWHM as function of $\chi$ is observed for the $131 \mathrm{~nm}$ sample, indicating the presence of significant inplane lattice disorder. It is important to note that the measured twist corresponds to the contributions of all grains pertaining to one of the fours twisted twin families, as schematically shown in Figure $6(b)$. By using Equation (4) and neglecting size effects (see Figure 3(a)) it is possible to obtain an excellent fit with values of $\Gamma_{y}=1.3^{\circ}$ and $\Gamma_{Z}=2.5^{\circ}$.

Another way to assess the average twist with respect to the perfect twin orientation is the evaluation of plan view TEM images. For this purpose, a plan view specimen of the $90 \mathrm{~nm} \mathrm{Mg} \mathrm{M}_{2}$ film of our series was prepared. Although preparation of such thin specimens is difficult by focus ion beam sectioning, especially because thinning needs to be performed from both sides in order to remove the substrate as well as the $\mathrm{MgO}$ capping layer, we chose this thin sample in order to exclusively observe the $\mathrm{Mg}_{3} \mathrm{~N}_{2}$ columns. The SAED pattern of the plan view specimen is presented in Figure 6(c). Besides confirming the epitaxial relationships discussed earlier (the $\mathrm{Mg}_{3} \mathrm{~N}_{2}(4 \overline{4} 0)$ reflection is aligned with the $\mathrm{MgO}(02 \overline{2})$ reflection), multiple diffraction spots are observed due to overlapping of the $\mathrm{Mg}_{3} \mathrm{~N}_{2}$ layer with the $\mathrm{MgO}$ substrate, which is still present in the specimen and whose diffraction is indicated by the dotted square. For the diffraction coming from $\mathrm{Mg}_{3} \mathrm{~N}_{2}$, a lateral elongation of the diffraction spots is observed, especially visible for the $\{440\}$ spots, indicating an average twist of $4-5^{\circ}$ between the columns. Although this measured twist seems much larger than previously determined by $\operatorname{XRD}\left(\Gamma_{z}=2.5^{\circ}\right)$, the two values are consistent with each other, given that XRD analysis provides the FWHMs of the peaks while the entire diffraction spot width was measured in TEM.

\section{GROWTH MODEL}

In the kinematical scattering theory, the diffracted intensity of a distorted crystal can be represented as the Fourier transform ${ }^{22}$ 


$$
I(\boldsymbol{q})=\int e^{i \boldsymbol{q} \cdot \boldsymbol{r}} G(\boldsymbol{r}) d \boldsymbol{r}
$$

of the correlation function

$$
G(\boldsymbol{r})=\langle\exp [i \boldsymbol{Q} \cdot(\boldsymbol{U}(\boldsymbol{r})-\boldsymbol{U}(0))]\rangle
$$

where $\boldsymbol{q}$ is a small deviation of $\boldsymbol{Q}$ with respect to the closest reciprocal lattice vector $\boldsymbol{Q}_{\mathbf{0}}$, and $\boldsymbol{U}(\boldsymbol{r})$ is the sum of all displacements produced by defects at a point $\boldsymbol{r}$. The exact nature of $G(\boldsymbol{r})$ depends strongly on the type and arrangement of the defects present in the layer.

Starting from Equation (8), Kaganer et. al constructed proper $G(\boldsymbol{r})$ functions that allowed them to identify types and arrangements of dislocations by simulating XRD patterns in a number of different heteroepitaxial systems ${ }^{11,16,18,19,24}$. In our case, a regular columnar structure was revealed by TEM, where the columns correspond to different twin domains that are correlated in the initial growth stages and lead to the narrow rocking curves. Nevertheless, there is an in-plane twist component present in the twins with respect to their ideal orientation, even in initial growth stages, causing the loss of coherent scattering in the XRD rocking curves of asymmetric reflections and leading to peak elongation in plan view SAED (indeed, no coherent peak was observed for any asymmetric reflection). As growth proceeds, the columns lose their correlation due to the introduction of additional misorientation accompanied by some overall relaxation of the films. This process is accompanied by the increase of diffuse scattering in XRD. Cross section TEM revealed that the tilt (based on Fourier transform images) increases as a function of thickness. Hence, the films consist of a two-layer structure that is schematically illustrated in Figure 7.

A similar columnar structure was recently found by Miyake et al. ${ }^{37,38}$ for sputtered AIN films grown on c-plane sapphire. The authors observed a $10 \mathrm{~nm}$ AIN nucleation layer consisting of columns with a diameter in the order of a few $\mathrm{nm}$, well-oriented along the [0001] growth direction, and displaying an in-plane twist of around $2^{\circ}$, giving rise also to two-component XRD rocking curves. Ca. $25 \mathrm{~nm}$ wide columns that are tilted with respect to the growth direction subsequently overgrow this nucleation layer, as observed in our thin films (though for different thicknesses). 
Furthermore, the large twist distributions observed in both materials systems might be related to the very-large lattice mismatches, which amount to $18 \%$ for the $\mathrm{Mg}_{3} \mathrm{~N}_{2}$ (111)-on-MgO (100) system and to $13 \%$ for the AIN on c-plane sapphire system. Indeed, Mante et al. ${ }^{39}$ developed a model explaining the twist during the nucleation stage of highly-mismatched materials systems and whose twist value is proportional to the initial lattice mismatch. To illustrate it experimentally, they analyzed the nucleation of AIN (0001) islands on $\mathrm{Si}(111)$ substrates, which is a material system displaying a $19 \%$ lattice mismatch. The TEM investigations revealed $60^{\circ}$ misfit dislocations at the film-substrate interface that compensated for the lattice parameter mismatch and included a $3.3^{\circ}$ twist between the nucleating islands as measured by grazing incidence XRD. Given the similar mismatch for the current $\mathrm{Mg}_{3} \mathrm{~N}_{2}$ (111)-on-MgO (100) system, it is possible that the observed twist distribution is inherent to the film/substrate combination that we have studied. Interestingly, in the two works on AIN strategies to improve the films quality were proposed. Mante et al. grew the nucleation layer at low temperature and increase the growth temperature for coalescence, whereas Miyake et al. used post-growth annealing at $1700{ }^{\circ} \mathrm{C}$ in order to coalesce their layers and annihilate many of the structural defects building up the grain boundaries. Such a post-growth treatment could be imaginable for our layers, too, although the actual presence of four twin domain families might render coalescence more difficult. Therefore, strategies to suppress the occurrence of epitaxial twin domains during growth, by means of symmetry breaking (e.g. by introducing a voluntarily misoriented substrate or by using other substrate orientations) should be employed to improve the film quality.

Although we achieved a good understanding of the nanostructure present in our $\mathrm{Mg}_{3} \mathrm{~N}_{2}$ films, the exact atomic structure of the twin boundaries remains unknown. Indeed, the overlap of several twins in cross section TEM prevented its exact determination. Numerical simulations as performed by Kaganer et al. may help to derive appropriate models and clarify the twin boundary structure on the atomic scale. 


\section{CONCLUSIONS}

A thorough structural characterization by means of HRXRD and TEM has enabled to establish a general picture of the microstructure of epitaxial $\mathrm{Mg}_{3} \mathrm{~N}_{2}$ thin films. The symmetry mismatch between the [111]oriented films (three-fold symmetry) and the $\mathrm{MgO}(100)$ substrate (four-fold symmetry) results in four twin domains, which arrange themselves in the form of $\sim 10 \mathrm{~nm}$ diameter columnar grains. $\mathrm{An} \mathrm{Mg}_{3} \mathrm{~N}_{2}$ layer displaying in-plane and out-of-plane rotational distributions several degrees wide, which degrade as the film thickness increases, overgrows these grains. Overall, this study demonstrates that epitaxial $\mathrm{Mg}_{3} \mathrm{~N}_{2}$ films can be obtained by plasma assisted molecular beam epitaxy on MgO substrates, but that their structural quality is still far from that of more mature semiconductors (e.g. GaN).

Thus, while a first step towards the introduction of $\mathrm{Mg}_{3} \mathrm{~N}_{2}$ as a new epitaxial semiconductor material has been realized, there is still a long way for its implementation in optoelectronic devices. This might require the growth optimization on different $\mathrm{MgO}$ crystallographic orientations or different substrates, to prevent the formation of twins, or the use of high-temperature annealing, which might induce grain coalescence and reduction of the defect density, as demonstrated recently in other materials (AIN) displaying a similar microstructure.

\section{SUPPLEMENTARY MATERIAL}

The supplementary material provides additional experimental data. It includes X-ray reflectivity curves, enabling to determine the thickness of the four thinnest thin films (Figure S1), and $\omega$ scans on the $\mathrm{Mg}_{3} \mathrm{~N}_{2}$ (222) symmetric reflection, illustrating the effect of the x-ray diffraction detection configuration on the measured FWHM for both the coherent and the diffuse contributions (Figure S2). It also contains RHEED and TEM images of the capping MgO layer (Figure S3), which show illustrate its polycrystalline nature, as well as RHEED images of a $\mathrm{Mg}_{3} \mathrm{~N}_{2}$ thin film at different growth stages (Figure S4). 


\section{Acknowledgments}

We thank Martiane Cabie and Thomas Neisius for TEM preparation and measurements and acknowledge support from the Agence Nationale de la Recherche through the project ZONE (ANR-17CE24-0043-01). Further, we thank Vladimir Kaganer (Paul Drude Institut, Berlin, Germany) for enlightening discussions and Mathieu Leroux (CRHEA, Valbonne, France) for his numerous suggestions.

\section{Data availability}

The data that support the findings of this study are available from the corresponding author upon reasonable request. 


\section{Figure Captions}

FIG. 1: (a) High-resolution $2 \theta / \omega$ scans of $\mathrm{Mg}_{3} \mathrm{~N}_{2}$ (222) reflections for films with different thicknesses, measured with a crystal analyzer detector (the diffraction curves are normalized and vertically shifted for clarity). The numerical fits are indicated by black dotted lines. The inset shows the (222) lattice spacings as a function of thickness resulting from the fits. (b) Normalized high-resolution (222) transverse $\omega$ scans for $\mathrm{Mg}_{3} \mathrm{~N}_{2}$ films of different thicknesses (measured with the same detector configuration as in (a)). The inset shows the normalized transverse $\omega$ scans of the $15 \mathrm{~nm} \mathrm{Mg} \mathrm{N}_{2}$ film and the $\mathrm{MgO}$ substrate.

FIG. 2: Rocking curves of the symmetric (222) and (444) reflections (i.e. of consecutive diffraction orders) measured with a $1 / 4^{\circ}$ slit in the diffraction path: (a) For a $69 \mathrm{~nm} \mathrm{Mg}_{3} \mathrm{~N}_{2}$ film, plotted as a function of in-plane reciprocal space units $Q_{x}$. (b) For a $302 \mathrm{~nm} \mathrm{Mg} \mathrm{M}_{2}$ film, plotted as a function of angular units $\omega$. The inset in (b) shows the same curves, but plotted as a function of in-plane reciprocal space units $Q_{x}$. The FWHM of the (222) and (444) reflection is constant for (a) and (b), as a function of $Q_{x}$ and $\omega$, respectively.

FIG. 3: (a) Goodness parameter $\Delta$ as a function of $\mathrm{Mg}_{3} \mathrm{~N}_{2}$ film thickness using Equation (3). The grey region indicates the critical thickness, for which the transition from a finite-size limited to a tilt limited structural quality occurs. (b) Diffraction tail analysis for the $\mathrm{Mg}_{3} \mathrm{~N}_{2}$ (444) reflection as a function of $\mathrm{Mg}_{3} \mathrm{~N}_{2}$ film thickness in log-log scale measured with a fully open detector.

FIG. 4: (a) Cross section TEM image of the $302 \mathrm{~nm}$-thick $\mathrm{Mg}_{3} \mathrm{~N}_{2}$ film along an $\mathrm{MgO}\langle 011\rangle$ zone axis. The $\mathrm{Mg}_{3} \mathrm{~N}_{2}$ (111) film displays a columnar structure close to the interface with the $\mathrm{MgO}(100)$ substrate that disappears at higher film thicknesses. (b) High-resolution TEM micrograph of the $\mathrm{MgO} / \mathrm{Mg}_{3} \mathrm{~N}_{2}$ substrate-layer interface. Insets $A$ to $D$ show Fourier transforms of high-resolution images taken at the corresponding positions indicated in (a).

FIG. 5: (a) Cross section SEAD pattern confirming the presence of four epitaxial twin domains as identified previously ${ }^{7}$ (b) Schematic representation of the four epitaxial $\mathrm{Mg}_{3} \mathrm{~N}_{2}(111)$ twins on $\mathrm{MgO}$ (100). The dashed line indicates the MgO [011] direction along which the sample preperation was performed. (c) Dark field image of the $302 \mathrm{~nm} \mathrm{Mg} \mathrm{N}_{2}$ (111) film (grown at $400{ }^{\circ} \mathrm{C}$ ), using $g=(044)$ of Twin I. Columns with an average height of ca. $70 \mathrm{~nm}$ are identified and can be associated to the different twin domains. (d) and (e) show the same experiments performed on a $97 \mathrm{~nm} \mathrm{Mg} \mathrm{N}_{2}$ (111) film grown at higher growth temperature $\left(460{ }^{\circ} \mathrm{C}\right)$, showing that the column height can be controlled by varying the growth parameters. Note that the specimen thickness is in the order of several tens of nanometers, leading to a superposition of several $\mathrm{Mg}_{3} \mathrm{~N}_{2}$ grains and preventing the observation of perfect complementary images. 
FIG. 6: (a) FWHMs of asymmetric reflections as a function of inclination angle for the $131 \mathrm{~nm}$ sample, measured with an open detector configuration. The points can be successfully fitted using the equation indicated in the figure and allowing the extraction of $2.5^{\circ}$ twist present in each epitaxial twin famiy. The inset shows the rocking curve of the asymmetric $\mathrm{Mg}_{3} \mathrm{~N}_{2}(044)$ reflection together with its fit, indicated by the red dotted line. (b) Schematic representation of a single twisted twin domain. (c) Plan view SAED pattern of the $90 \mathrm{~nm}$ sample. Diffraction spots coming from the $\mathrm{MgO}$ substrate are indicated by the dotted square.

FIG. 7: Schematic representation of the two-layer structure found in our $\mathrm{Mg}_{3} \mathrm{~N}_{2}$ films. The first $\sim 70 \mathrm{~nm}$ show a regular, columnar structure consisting of epitaxial twin domains, which are well aligned along the [111] growth direction, but exhibit an in-plane twist component. As growth proceeds, correlation is lost and additional disorder (both tilt and twist) is introduced into the film. 


\section{References}

${ }^{1}$ A. David and L.A. Whitehead, C. R. Phys. 19, 169 (2018).

${ }^{2}$ M.T. Hardy, D.F. Feezell, S.P. DenBaars, and S. Nakamura, Mater. Today 14, 408 (2011).

${ }^{3}$ T.J. Flack, B.N. Pushpakaran, and S.B. Bayne, J. Electron. Mater. 45, 2673 (2016).

${ }^{4}$ J. Zúñiga-Pérez, V. Consonni, L. Lymperakis, X. Kong, A. Trampert, S. Fernández-Garrido, O. Brandt, H. Renevier, S. Keller, K. Hestroffer, M.R. Wagner, J.S. Reparaz, F. Akyol, S. Rajan, S. Rennesson, T. Palacios, and G. Feuillet, Appl. Phys. Rev. 3, 041303 (2016).

${ }^{5}$ Y. Uenaka and T. Uchino, J. Phys. Chem. C 118, 11895 (2014).

${ }^{6}$ C.M. Fang, R.A. de Groot, R.J. Bruls, H.T. Hintzen, and G. de With, J. Phys. Condens. Matter 11, 4833 (1999).

${ }^{7}$ P. John, H. Rotella, C. Deparis, G. Monge, F. Georgi, P. Vennéguès, M. Leroux, and J. Zuniga-Perez, Phys. Rev. Mat. 4, 054601 (2020).

${ }^{8}$ P. Wu, X. Cao, T. Tiedje, and N. Yamada, Mater. Lett. 236, 649 (2019).

${ }^{9}$ P. Wu and T. Tiedje, Appl. Phys. Lett. 113, 82101 (2018).

${ }^{10}$ C.G. Darwin, Philos. Mag. 27, 675 (1914)

${ }^{11}$ V.M. Kaganer, O. Brandt, A. Trampert, and K.H. Ploog, Phys. Rev. B 72, 45423 (2005).

${ }^{12}$ T. Metzger, R. Höpler, E. Born, S. Christiansen, M. Albrecht, H.P. Strunk, O. Ambacher, M. Stutzmann, R. Stömmer, M. Schuster, and H. Göbel, Phys. Status Solidi A 162, 529 (1997).

${ }^{13}$ M.W. Cho, A. Setaiwan, H.J. Ko, S.K. Hong, and T. Yao, Semicond. Sci. Technol. 20, S13-S21 (2005).

${ }^{14}$ J. Bläsing, A. Krost, J. Hertkorn, F. Scholz, L. Kirste, A. Chuvilin, and U. Kaiser, J. Appl. Phys. 105, 33504 (2009).

${ }^{15}$ M. Becht, F. Wang, J.G. Wen, and T. Morishita, J. Crys. Growth 170, 799 (1997).

${ }^{16}$ V.M. Kaganer, O. Brandt, H. Riechert, and K.K. Sabelfled, Phys. Rev. B 80, 33306 (2009).

${ }^{17}$ A. Boulle, R. Guinebretière, and A. Dauger, J. Appl. Phys. 97, 73503 (2005).

${ }^{18}$ V.M. Kaganer, R. Köhler, M. Schmidbauer, and R. Opitz, Phys. Rev. B 55, 1793 (1997).

${ }^{19}$ V.M. Kaganer and K.K. Sabelfeld, Phys. Rev. B 80, 184105 (2009).

${ }^{20}$ O. Durand, A. Letoublon, D.J. Rogers, and F. Hosseini Teherani, Thin Solid Films 519, 6369 (2011).

${ }^{21}$ R.I. Barabash, W. Donner, and H. Dosch, Appl. Phys. Lett. 78, 443 (2001).

${ }^{22}$ P.F. Miceli and C.J. Palstrøm, Phys. Rev. B 51, 5506 (1995).

${ }^{23}$ P.F. Miceli, J. Weatherwax, T. Krentsel, and C.J. Palstrøm, Physica B Condens. Matter 221, 230 (1996).

${ }^{24} \mathrm{~V}$. Kaganer, T. Ulyanenkova, A. Benediktovitch, M. Myronov, and A. Ulyanenkov, J. Appl. Phys. 122, 105302 (2017).

${ }^{25}$ H. Heinke, V. Kirchner, H. Selke, R. Chierchia, R. Ebel, and S. Einfeldt, J. Phys. D: Appl. Phys. 34, A25 (2001). 
${ }^{26}$ D.R. Glasson and S.A. A. Jayaweera, J. Appl. Chem. 18, 77 (1968).

${ }^{27}$ X.D. Peng, D.S. Edwards, and M.A. Barteau, Surf. Sci. 195, 103 (1988).

${ }^{28}$ A.M. Heyns, L.C. Prinsloo, K.J. Range, and M. Stassen, J. Sol. Stat. Chem. 137, 33 (1998).

${ }^{29}$ J.-M. Triscone, P. Fivat, M. Andersson, M. Decroux, and $\varnothing$. Fischer, Phys. Rev. B 50, 1229 (1994).

${ }^{30} \mathrm{C}$. Lichtensteiger, J. Appl. Crystallogr. 51, 1745 (2018).

${ }^{31}$ T. Böttcher, S. Einfeldt, S. Figge, R. Chierchia, H. Heinke, D. Hommel, and J.S. Speck, Appl. Phys. Lett. 78, 1976 (2001).

${ }^{32}$ B.W. Sheldon, K.H.A. Lau, and A. Rajamani, J. Appl. Phys. 90, 5097 (2001).

${ }^{33}$ J.A. Floro, E. Chason, R.C. Cammarata, and D.J. Srolovitz, MRS Bull. 27(1), 19-25 (2002).

${ }^{34}$ G.K. Williamson and W.H. Hall, Acta Metall., 1, 22 (1953).

${ }^{35}$ M. Grundmann, Phys. Stat. Sol. B 248, 805 (2011).

${ }^{36}$ S.R. Lee, A.M. West, A.A. Allermann, K.E. Waldrip, D.M. Follstaedt, P.P. Provencio, and D.D. Koleske, Appl. Phys. Lett. 86, 241904 (2005).

${ }^{37}$ H. Miyake, C.-H. Lin, K. Tokoro, and K. Hiramatsu, J. Crys. Growth 456, 155 (2016).

${ }^{38}$ S. Xiao, R. Suzuki, H. Miyake, S. Harada, and T. Ujihara, Journal of Crystal Growth 502, 41 (2018).

${ }^{39}$ N. Mante, S. Rennesson, E. Frayssinet, L. Largeau, F. Semond, J.L. Rouvière, G. Feuillet, and P. Vennéguès, J. Appl. Phys. 123, 215701 (2018). 

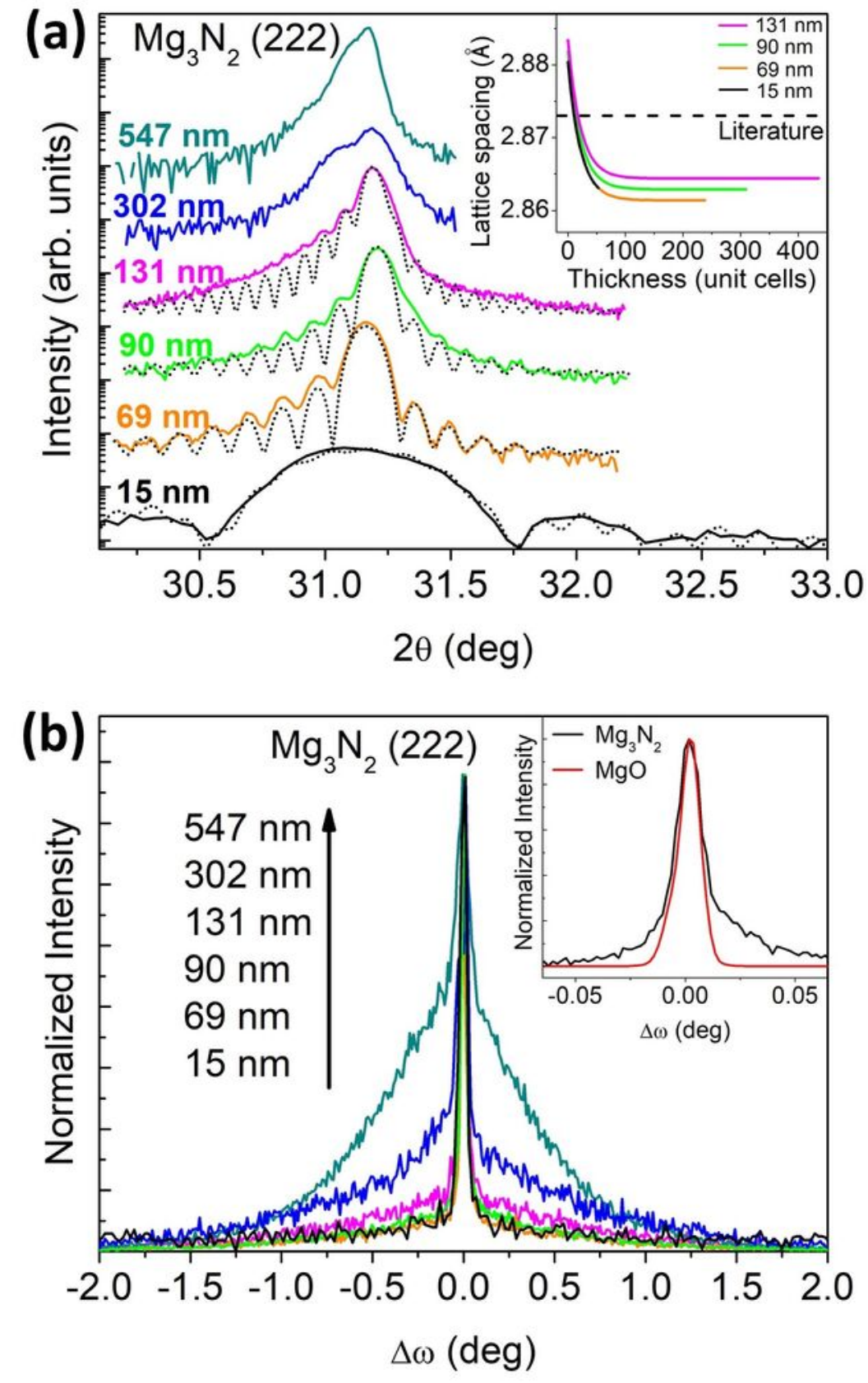

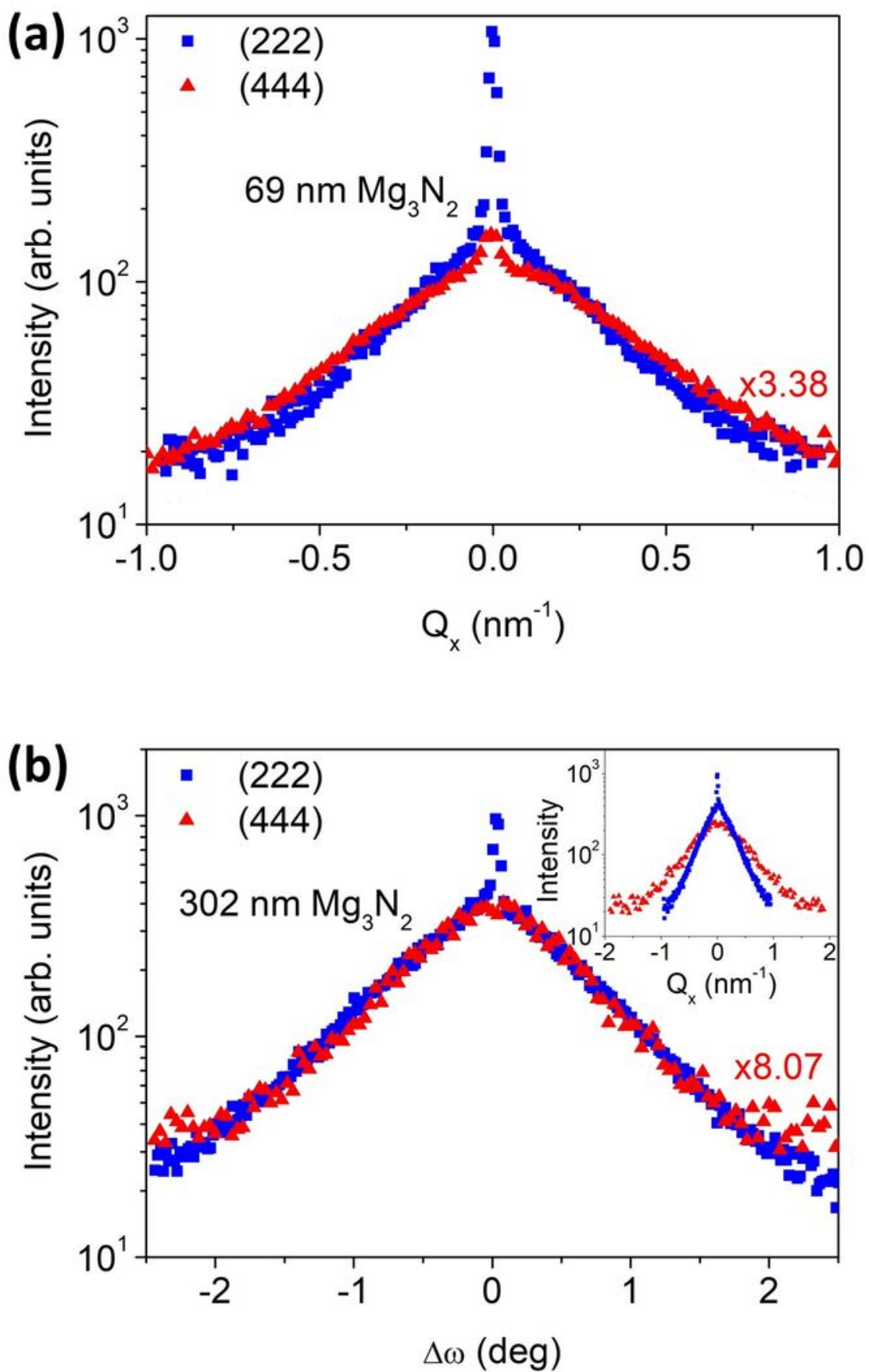

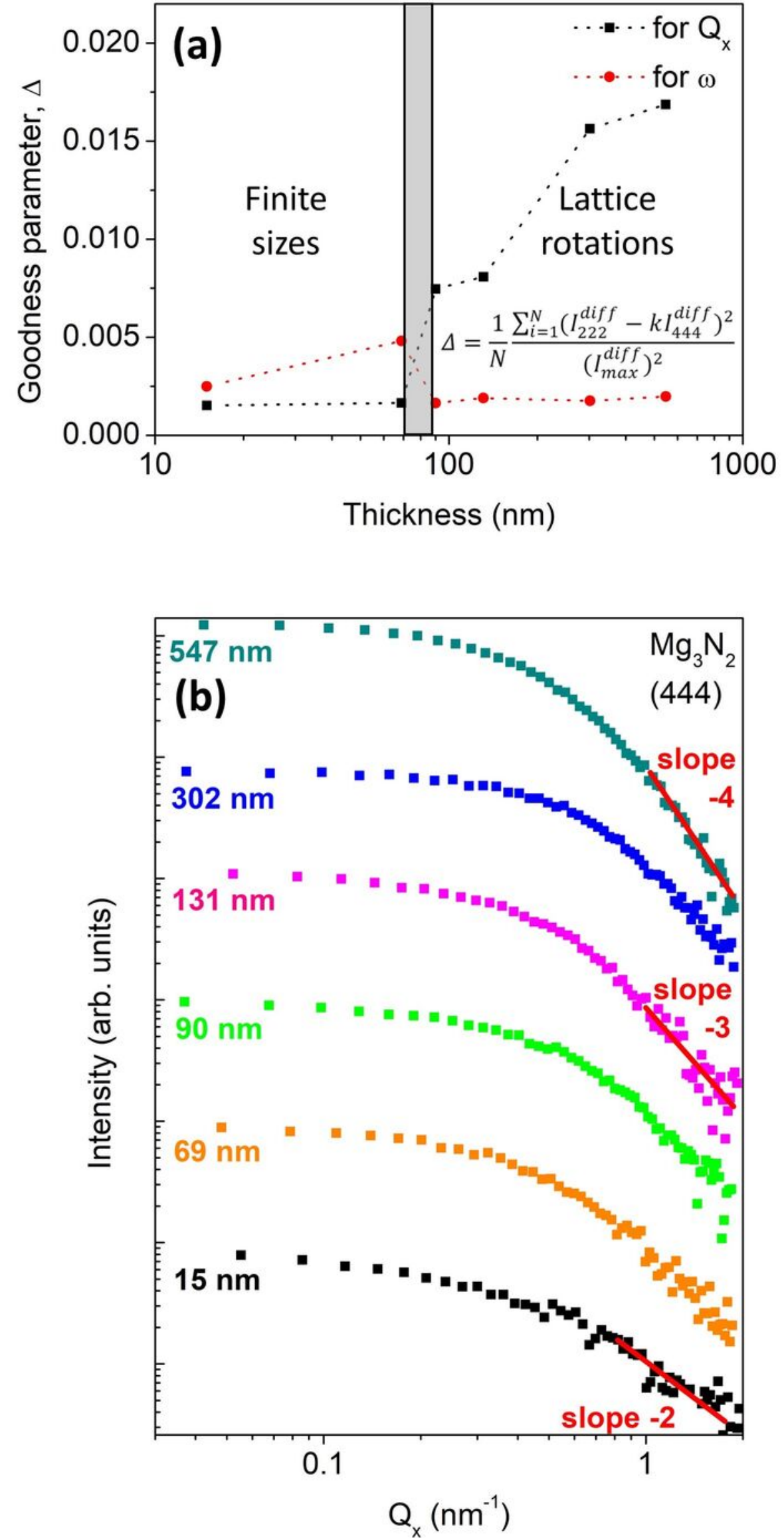


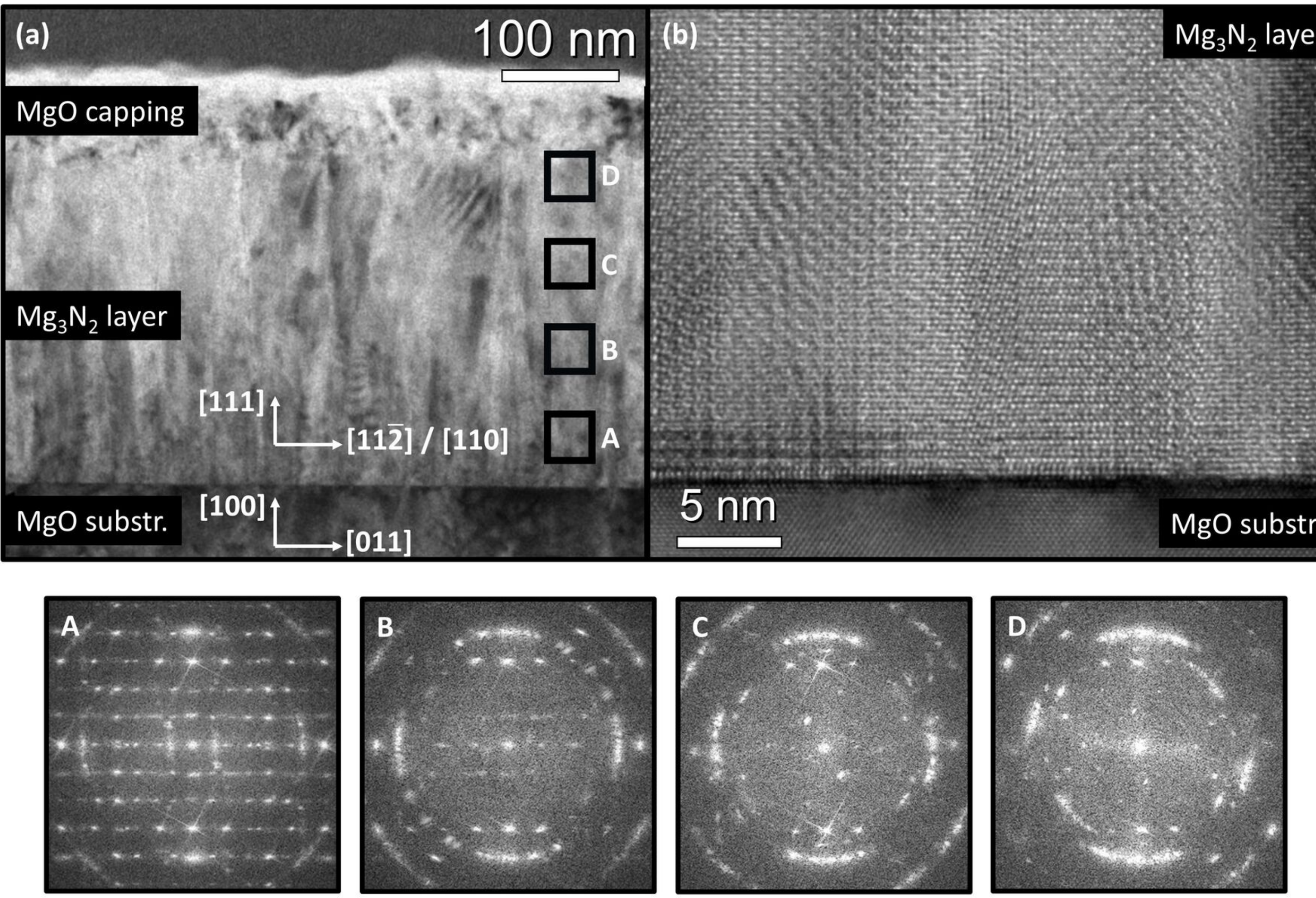




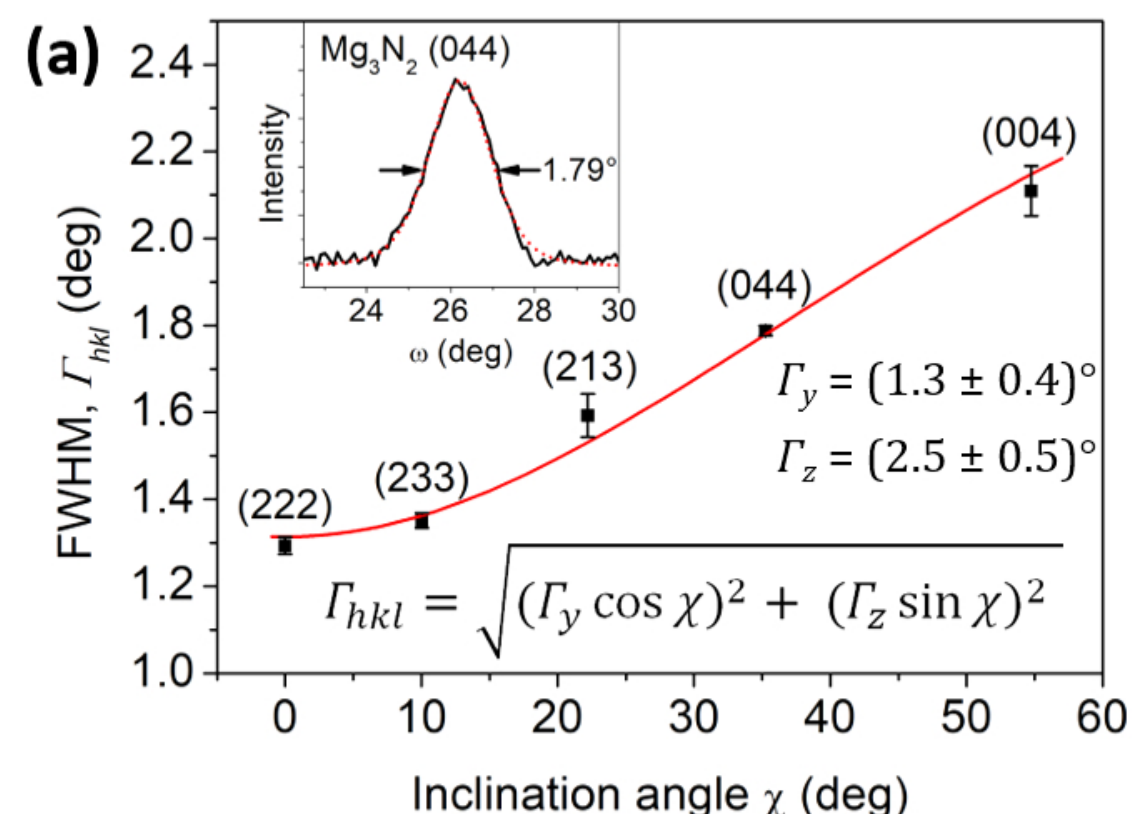

(b)

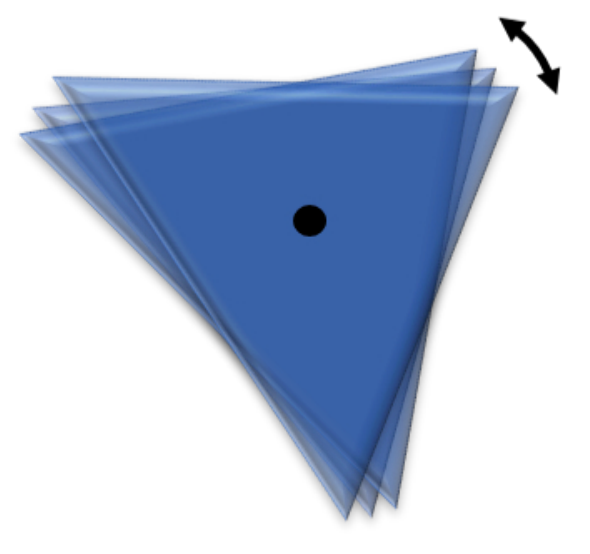

Twist in one twin family

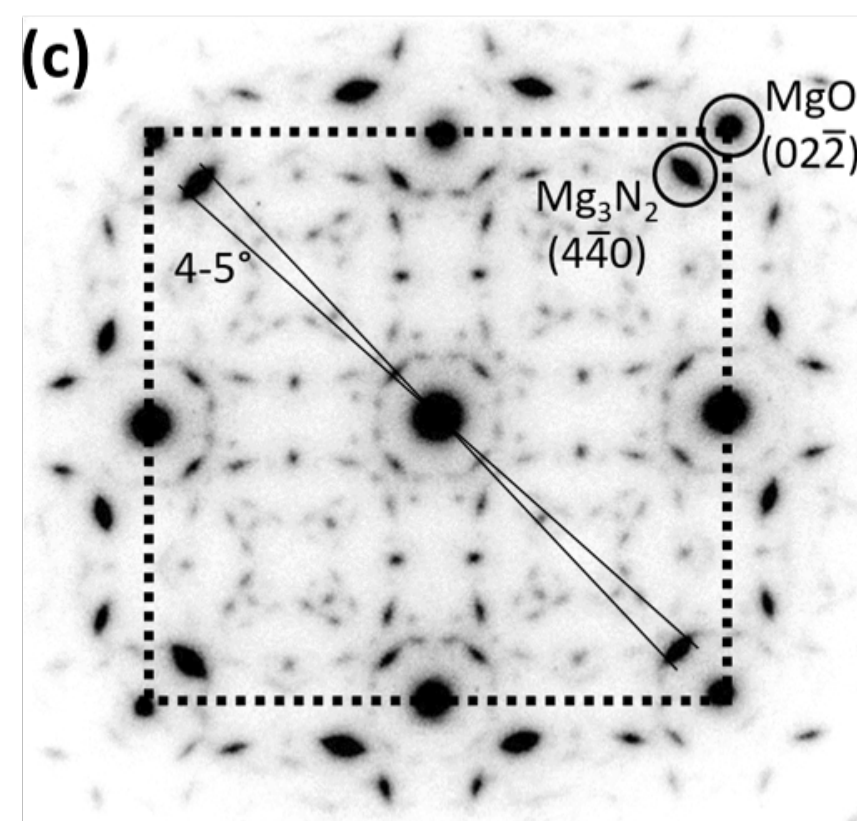




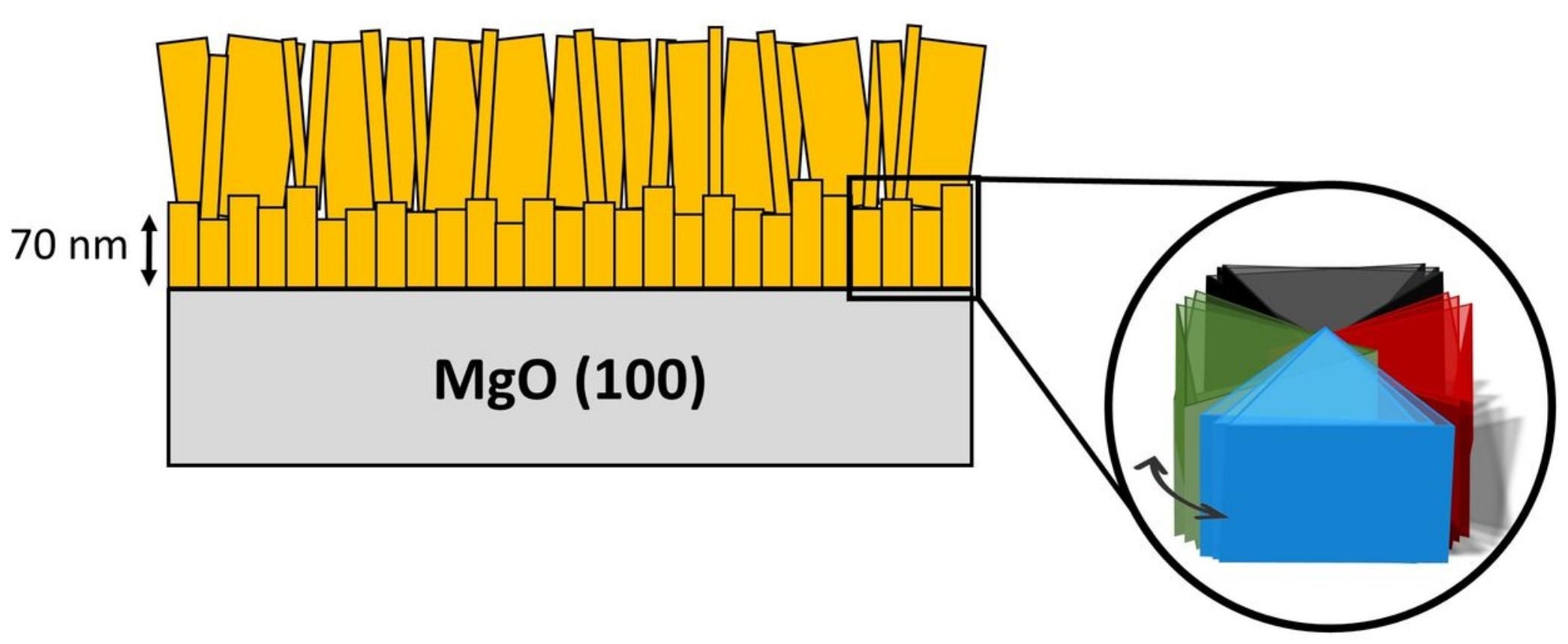

